\title{
DESIGN OF COLUMNS CENTRICALLY LOADED IN COMPRESSION
}

Design of centrically loaded columns by the axial compressive load is considered in this work. The three methods results are compared: theoretical w-procedure and calculations according to standards JUS U.E7.081and Eurocode 3. The analysis is illustrated on an example of a column with a complex cross-section. By comparison between the calculated normal stresses and should be allowable stresses for all the three procedures, one can see that the stresses calculated using both standard procedures are significantly smaller than stresses calculated using the theoretical procedure. This shows that the standard procedures of calculations and design are much more on the safety side, especially in the case of Eurocode 3.

\section{Introduction}

The columns loaded in compression must be checked for stability, i.e., against the possibility of buckling. There exist numerous methods for these calculations; from the classical Eulerian procedure, through Engesser - Karman corrections, to the application of generally adopted international standards. The intention here is to compare the three design (calculations) procedures, one theoretical, the so-called $\omega$-procedure, and the two standards' prescribed procedures, by the JUS and Eurocode 3. It will be shown which procedure gives the best estimate of the load carrying capacity of the compressed column and which procedure(s) are on the safety side and for how much.

\section{The design of columns centrically loaded by compressive load}

Load-carrying capacity of axially loaded columns, by using the w-procedure, is written as, [1-3]:

$$
\sigma_{\omega}=\frac{N}{A} \omega \leq \sigma_{d o p},
$$

where coefficient $\omega$ depends on effective slenderness ratio, $\lambda$.

Load-carrying capacity of axially loaded columns, with a singlepart cross-section, by using the standard procedure JUS U. E7. 081/198 [4], is written as:

$$
\sigma_{N}=\frac{N}{A} \leq \sigma_{i, d o p}=\chi \sigma_{d o p},
$$

where: $N$ is the calculated normal force for appropriate case of loading, $A$ is the cross-sectional area, $\sigma_{N}$ is the calculated normal stress, $\sigma_{i, d o p}$ is the allowable buckling stress, $\sigma_{d o p}$ is the allowable normal stress, $\chi$ is the buckling reduction factor, which depends on relative slenderness $\bar{\lambda}$, shape of cross-section and degree of equivalent geometric imperfections.

Dependence of the column's cross section and degree of equivalent geometric imperfections are expressed by column belonging to one of the buckling curves, $A_{0}, A, B, C$ or $D$, Figure 1, [4].

Relative slenderness ratio $\bar{\lambda}$ is the relation between the effective slenderness ratio $\lambda$ and slenderness ratio at yield strength, $\lambda_{v}$ :

$$
\bar{\lambda}=\lambda / \lambda_{v}
$$

Effective slenderness ratio is a quotient of the effective length of the column, $\ell_{k i}$ and competent radius of gyration $i$ :

$$
\lambda=\ell_{k i} / i
$$

Load-carrying capacity of axially loaded columns, with a singlepart cross-section, according to Eurocode 3, should be verified against buckling as follows, [5]:

$$
\frac{N_{E d}}{N_{b, R d}} \leq 1.0,
$$

where: $N_{E d}$ is the design value of the compression force; $N_{b, R d}$ is the design buckling resistance of the compression member.

For members with non-symmetric Class 4 sections allowance should be made for the additional moment $\Delta M_{E d}$ due to the eccentricity of the centroidal axis of the effective section.

\footnotetext{
* Ruzica R. Nikolic ${ }^{1}$, Jelena M. Veljkovic ${ }^{2}$, Jozef Vican ${ }^{3}$

${ }^{1}$ Faculty of Mechanical Engineering, University of Kragujevac, Sestre Janjic 634000 Kragujevac, Serbia,

E-mail: ruzicarnikolic@yahoo.com and inikolic@ptt.yu

${ }^{2}$ Executive research manager, “RAPP-ZASTAVA” Factory, Trg Topolivca 4, 34000 Kragujevac, Serbia, E-mail: vkatarina@ptt.yu

${ }^{3}$ Faculty of Civil Engineering, University of Zilina, Komenskeho 52, 01026 Zilina, Slovakia, vican@fstav.uniza.sk
} 


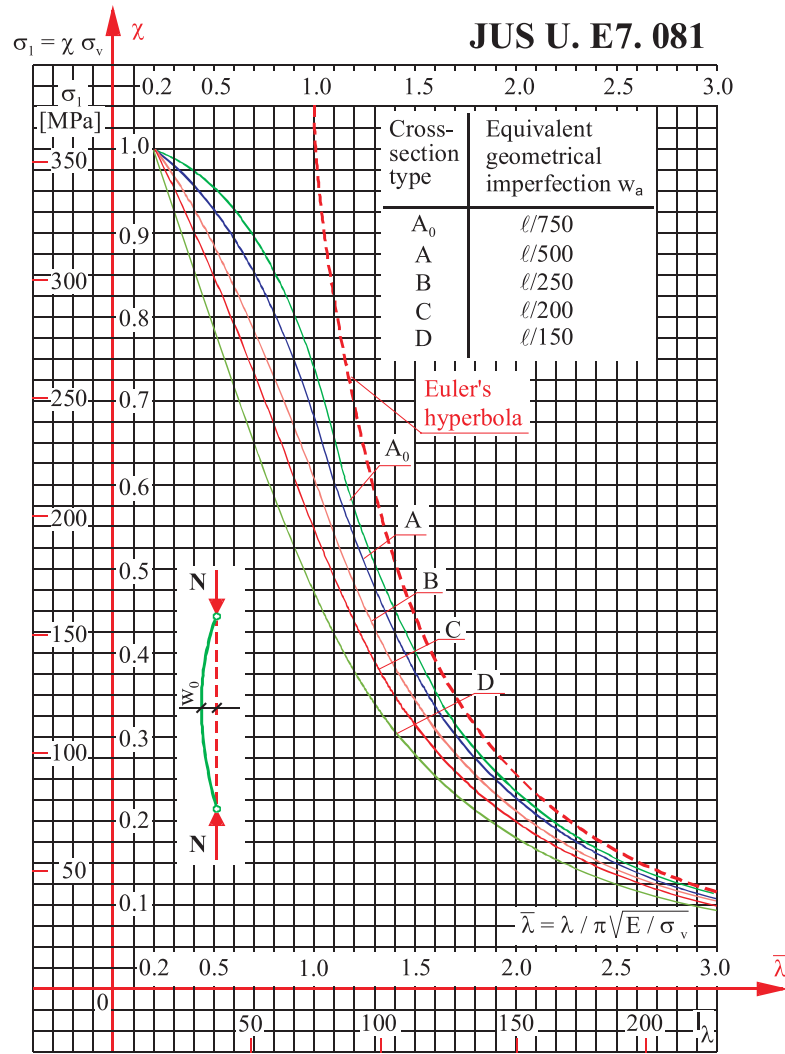

Fig. 1 Buckling curve according to the standard procedure JUS U. E7. 081/198

The design buckling resistance of a compression member should be taken as for Class 1, 2 and 3 cross-sections

$$
N_{b, R d}=\chi A f_{y} / \gamma_{M 1}
$$

and for Class 4 cross-sections:

$$
N_{b, R d}=\chi A_{e f f} f_{y} / \gamma_{M 1},
$$

where $\chi$ is the reduction factor for the relevant buckling mode, $f_{y}$ is the yield stress and $\gamma_{M 1}$ is partial factor for resistance to instability.

For axial compression in members, the value of $\chi$ for the appropriate non-dimensional slenderness $\bar{\lambda}$ should be determined from the relevant buckling curve according to

$$
\chi=1 /\left(\Phi+\sqrt{\Phi^{2}-\bar{\lambda}^{2}}\right),
$$

but $\chi \leq 1.0$, where $\Phi=0.5\left\lfloor\left[1+\alpha(\bar{\lambda}-0.2)+\bar{\lambda}^{2}\right]\right\rfloor$ with:

$$
\bar{\lambda}=\sqrt{A_{f y} / N_{c r}} \text { and } \bar{\lambda}=\sqrt{A_{e f f} f_{y} / N_{c r}},
$$

for the Class 1, 2 and 3 and for the Class 4 cross-sections, respectively, where: $\alpha$ is an imperfection factor. The imperfection factor $\alpha$ corresponding to the appropriate buckling curve should be obtained from Tables 1 and 2.

Imperfection factor for different

Table 1.

buckling curves (Table 6.1, [5])

\begin{tabular}{|l|c|c|c|c|c|}
\hline Buckling curve & $\mathrm{a}_{0}$ & $\mathrm{a}$ & $\mathrm{b}$ & $\mathrm{c}$ & $\mathrm{d}$ \\
\hline Imperfection factor $\alpha$ & 0.13 & 0.21 & 0.34 & 0.49 & 0.76 \\
\hline
\end{tabular}

Buckling curves for various

Table 2 . cross-sections (Table 6.2, [5])

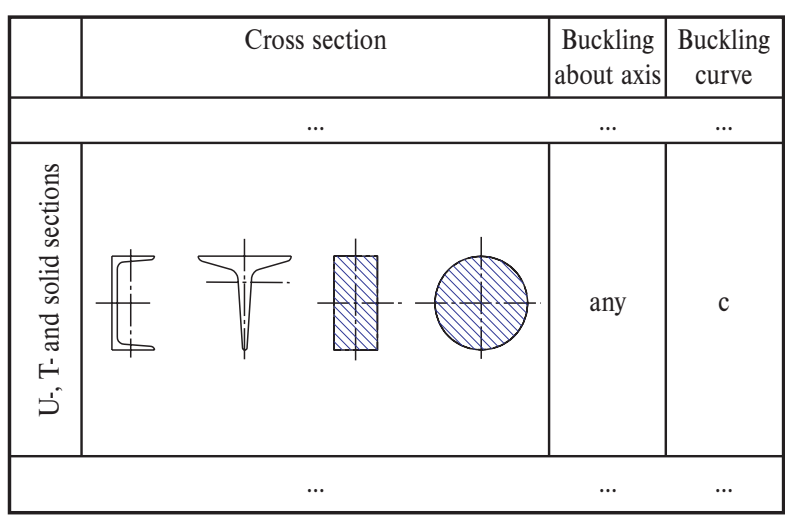

$N_{c r}$ is the elastic critical force for the relevant buckling mode based on the gross cross sectional properties.

Values of the reduction factor $\chi$ for the appropriate non-dimensional slenderness $\bar{\lambda}$ may be obtained from Figure 2 .

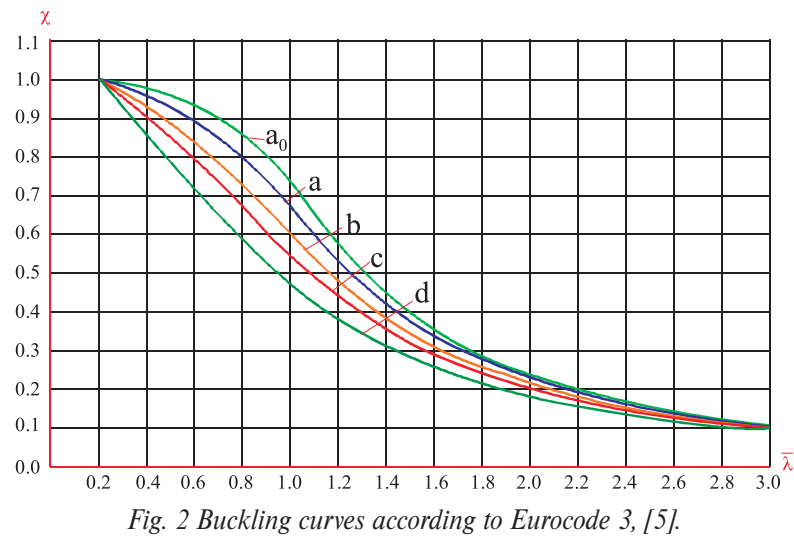

For slenderness $\bar{\lambda} \leq 0.2$ or for $N_{E d} / N_{c r} \leq 0.04$ the buckling effects may be ignored and only cross sectional checks apply.

The non-dimensional slenderness $\bar{\lambda}$ is given by:

$\bar{\lambda}=\sqrt{A f_{y} / M}=\left(L_{c r} / i\right)\left(1 / \lambda_{1}\right)$ and 


$$
\bar{\lambda}=\sqrt{A_{e f f} f_{y} / N}=\left(L_{c r} / i\right)\left(\sqrt{\frac{A_{e f f}}{A}} / \lambda_{1}\right),
$$

for the Class 1, 2 and 3 and for the Class 4 cross-sections, respectively. Here, $L_{c r}$ is the buckling length in the buckling plane considered; $i$ is the radius of gyration about the relevant axis, determined using the properties of the gross cross-section

$$
\lambda_{1}=\pi \sqrt{E / f_{y}}=93.9 \epsilon ; \epsilon=\sqrt{235 / f_{y}},
$$

where $f_{y}$ is in $\mathrm{N} / \mathrm{mm}^{2}$.

\section{The design procedures for compressively centrically loaded columns comparison}

The cross section of the multi-part columns is characterized by the material and non-material (free) axes. The former crosses all the individual parts of the cross section, while the latter does not necessarily pass through all the parts of cross section. The lace sheets and truss bars are positioned perpendicular to the free axis.

The individual element axis is the axis that corresponds to the minimum gyration radius. The carrying capacity control of centrically compressed columns, of the constant, multi-part cross-section, enhances the control of buckling resistance both around the material and around non-material axes.

The problem of design centrically loaded column by compressive load will be illustrated on an example of a column with crosssection shown in Figure 3 (battened built-up members). The column height is $h=7 \mathrm{~m}$, and axial compressive force is $F=840 \mathrm{kN}$. The material is $\check{C} 0361$ (corresponding to S 275 in ENV 1993 [5]).

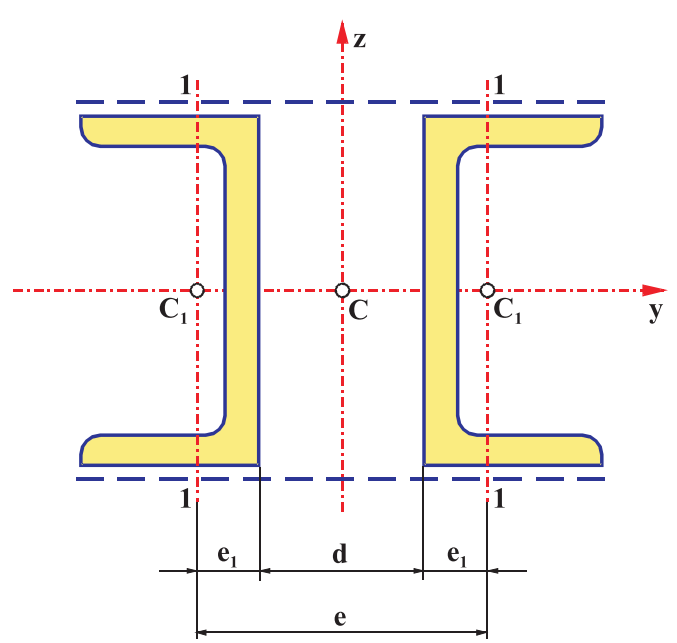

Fig. 3 Cross-section of a column

$\omega_{z_{1}}=2 \cdot A_{1} \cdot \sigma_{d o p} / F=1.61 \Rightarrow \lambda z_{i}=85,[6], \lambda_{1}=\frac{a}{i_{1}}=\frac{100}{2.42}=$ $=41.32 \approx 41,(a=1000 \mathrm{~mm}$ - distance between the two lacing bars, Figure 4).
In the first place, dimensions necessary to carry actual load, will be determined using the $\omega$-procedure [6,7].

Firstly, is selected $U 24$, with the following data (Figure 3):

$I_{y_{1}}=3600 \cdot 10^{4} \mathrm{~mm}^{4}, I_{z_{1}}=248 \cdot 10^{4} \mathrm{~mm}^{4}, A_{1}=4230 \mathrm{~mm}^{2}$,

$e_{1}=233 \mathrm{~mm}, i_{z_{1}}=i_{1}=24.2 \mathrm{~mm}, i_{y_{1}}=92.2 \mathrm{~mm}$

For $2 U 24$ column cross-section is:

$I_{y}=2 \cdot I_{y_{1}}=7200 \cdot 10^{4} \mathrm{~mm}^{4} ; i_{y}=\sqrt{I_{y} / A}=\sqrt{2 \cdot I_{y_{1}} / 2 \cdot A_{1}}=$

$=\sqrt{I_{y_{1}} / A_{1}}=i_{y_{1}}=92.2 \mathrm{~mm}$.

$\lambda_{y}=h / i_{y}=75.92 \Rightarrow \lambda_{y}=76 \Rightarrow \omega_{y}=1.46$, [4].

$\sigma_{\omega_{y}}=\left(F / 2 \cdot A_{1}\right) \cdot \omega_{y}=145 \mathrm{MPa} ;$

$\sigma_{\omega_{y}}=145 \mathrm{Mpa}<\sigma_{d o p}=160 \mathrm{MPa}$.

The $2 U 24$ cross-section is well selected in view of buckling stability around the $y$-y axis.

It is necessary to check validity of criterion of stability around the $z-z$ axis. Lattice $e$, namely, $d$ is unknown, and will be determined under condition that the material is utilized by buckling around that axis up to $\sigma_{d o p}=160 \mathrm{MPa}$, [4].

$$
\begin{aligned}
& \sigma_{\omega_{z}}=(F / A) \cdot \omega_{z_{i}}=\sigma_{d o p} \Rightarrow \omega_{z_{i}}=2 \cdot A_{1} \cdot \sigma_{d o p} / F=1.61 \Rightarrow \\
& \lambda_{z_{i}}=85,[6] . \\
& \lambda_{1}=a / i_{1}=41.32 \approx 41-\text { (Distance between the two lacing }
\end{aligned}
$$
bars is $a=1000 \mathrm{~mm}$ ).

$$
\begin{aligned}
& \lambda_{z_{i}}=\sqrt{\lambda_{z}^{2}+\lambda_{1}^{2}} \Rightarrow \lambda_{z}=\sqrt{\lambda_{z_{i}}^{2}-\lambda_{1}^{2}}=74.4 ; \lambda_{z}=\ell_{k z} / i_{z}= \\
& =h / i_{z} \Rightarrow i_{z}=h / \lambda_{z}=94.1 \mathrm{~mm} \\
& I_{z}=i_{z}^{2} \cdot A=7490 \cdot 10^{4} \mathrm{~mm}^{4} \quad ; \\
& I_{z}=2 \cdot\left(I_{z_{1}}+y_{c_{1}}^{2} \cdot A_{1}\right) \Rightarrow e^{2} \cdot\left(A_{1} / 2\right)=I_{z}-2 \cdot I_{z_{1}} \Rightarrow \\
& e=\sqrt{2 \cdot\left(I_{z}-2 \cdot I_{z y_{1}}\right) / A_{1}}=182 \mathrm{~mm} .
\end{aligned}
$$

For $e=182 \mathrm{~mm} \Rightarrow d=e-2 \cdot e_{1}=137.4 \mathrm{~mm}$, thus the value of $d=160 \mathrm{~mm}$ is adopted.

For $\mathrm{d}=160 \mathrm{~mm} \Rightarrow d=e+2 \cdot e_{1}=204.6 \Rightarrow$

$I_{z}=2 \cdot\left(248 \cdot 10^{4}+(204.6)^{2} \cdot 4230\right)=9340 \cdot 10^{4} \mathrm{~mm}^{4}$.

$i_{z}=\sqrt{I_{z} / A}=105 \mathrm{~mm} \Rightarrow \lambda_{\mathrm{z}}=\mathrm{h} / \mathrm{i}_{\mathrm{z}}=67$. 
Control of buckling stability around the $z-z$ axis:

$\lambda_{z_{i}}=\lambda_{z}^{2}+\lambda_{1}^{2}=79 \Rightarrow \omega_{z_{1}}=1.51,[6]$.

$\sigma_{\omega_{y}}=(F / A) \cdot \omega_{z_{1}}=150 \mathrm{MPa}<\sigma_{d o p}=160 \mathrm{MPa}$, thus the stability checks.

Control of the distance between the two lacing bars:

$$
\begin{aligned}
& \lambda_{1}=c / i_{1}=41 \leq 50 \cdot\left(4-3 \cdot\left(\sigma_{\omega} / \sigma_{\text {dop }}\right)\right)=59 \Rightarrow \\
& \Rightarrow \lambda_{1}=41<59
\end{aligned}
$$

The cross-section $2 U 24$ is selected with lattice $d=16 \mathrm{~cm}$.

In further analysis is illustrated a design of centrically compressed column according to the standard procedure JUS U. E7. 081.

The proof of load-carrying capacity around the material axis $y-y$, is as follows:

$$
\lambda_{i, y}=\ell_{i, y} / i_{y}=75.92 ; \quad \bar{\lambda}_{y}=\lambda_{i, y} / \lambda_{v}=0.82 \Rightarrow \chi=0.65,
$$
(Curve "C", Figure 1).

Allowable buckling stress is: $\sigma_{i, d o p}=\chi \cdot \sigma_{d o p}=104 \mathrm{MPa}$

Calculated normal stress is: $\sigma_{N}=N / A=99.3 \mathrm{MPa}$, thus the stability checks.

The proof of the load-carrying capacity around the nonmaterial axis $z-z$, is as follows:

$$
\lambda_{z}=\ell_{i, z} / i_{z}=67 \Rightarrow \lambda_{z i}=\sqrt{\lambda_{z}^{2}+(m / 2) \cdot \lambda_{1}^{2}}=78.55,
$$

with $m=2-$ number of single elements in a complex cross-section

$$
\lambda_{1}=\mathrm{c} / \mathrm{i}_{1}=41.32 \approx 41 ; \bar{\lambda}_{z i}=\frac{\lambda_{z i}}{\lambda_{v}}=0.85 \Rightarrow \chi=0.63 \text {, (Curve }
$$
"C", Figure 1).

Allowable buckling stress is: $\sigma_{i, d o p}=\chi \cdot \sigma_{d o p}=100.8 \mathrm{MPa}$, while the calculated normal stress is: $\sigma_{N}=N / A=99.3 \mathrm{MPa}$, thus the stability checks.

Finally, the dimensioning of the compressed columns according to Eurocode 3, [5] will be presented.

Checks should be performed for chords using the design chord forces $N_{c h, E d}$ from compression forces $N_{E d}$ and moments $M_{E d}$ at mid span of the built-up member.

For a member with the two identical chords the design force $N_{c h, E d}$ should be determined from:

$$
N_{c h, E d}=0.5 N_{E d}+\left(M_{E d} h_{0} A_{c h} / 2 I_{e f f}\right),
$$

where: $M_{E d}=\left(N_{E d} e_{0}+M_{E D}^{I}\right) /\left[1-\left(N_{E d} / N_{c r}\right)-\left(N_{E d} / S_{v}\right)\right] ; e_{0}=$ $=L / 500$ is the initial bow imperfection; $N_{c r}=\pi^{2} E I_{\text {eff }} / L^{2}$ is the effective critical force of the built-up member; $N_{E d}$ is the design value of the compression force to the built-up member; $M_{E d}$ is the design value of the maximum moment in the middle of the builtup member considering second order effects; $M_{E D}^{I}$ is the design value of the maximum moment in the middle of the built-up member without second order effects; $h_{0}$ is the distance between the centroids of chords, $h_{0}=204.6 \mathrm{~mm} ; A_{c h}$ is the cross-sectional area of one chord; $I_{\text {eff }}$ is the effective second moment of area of the built-up member; $S_{v}$ is the shear stiffness of the lacings or battened panel.

For chords the buckling verification should be performed as follows:

$$
\left(N_{c h, E d} / N_{b, R d}\right) \leq 1.0,
$$

where: $N_{c h . E d}$ is the design compression force in the chord at midlength of the built-up member and $N_{b, R d}$ is the design value of the buckling resistance of the chord taking the buckling length $L_{c h}$ from Figure 4.

The shear stiffness $\mathrm{S}_{\mathrm{V}}$ should be taken as:

$$
\left.S_{v}=\left\{24 E I_{c h} / a^{2}\left[1+2 I_{c h} / n I_{b}\right)\left(h_{0} / a\right)\right]\right\} \leq\left(2 \pi^{2} E I_{c h} / a^{2}\right) .
$$

The effective second moments of area of battened built-up members may be taken as:

$$
I_{e f f}=0.5 h_{0}^{2} A_{c h}+2 \mu I_{c h},
$$

where $I_{c h}$ is the in plane second moment of area of one chord, $I_{b}$ is the in plane second moment of area of one batten, $\mu$ is the efficiency factor from Table 3 .

Using the previously obtained data from the example, the calculations are as follows:

$$
\begin{aligned}
& e_{0}=L / 500=14 \mathrm{~mm} ; I_{e f f}=0.5 h_{0}^{2} A_{c h}+2 \mu I_{c h}= \\
& =93496353.4 \mathrm{~mm}^{2} \\
& N_{c r}=\pi^{2} E I_{e f f} / L^{2}=3950728.5 \mathrm{~N} ; S_{v}=2 \pi^{2} E I_{c h} / a^{2}= \\
& =10269759.36 \mathrm{~N} \\
& N_{b, R d}=\chi A_{c h} f_{y} / \gamma_{M I}=988762.5 \mathrm{~N} ; \bar{\lambda}=\sqrt{A_{c h} f_{y} / N_{c r}}= \\
& =\left(L_{c h} / i\right)\left(1+\lambda_{1}\right)=0.54 ; L_{c h}=a=1000 \mathrm{~mm} ; \\
& \lambda_{1}=\pi \sqrt{E / f_{y}}=93.9 \epsilon=76.06 \Rightarrow \epsilon=0.81 ; \chi=0.85 ; \\
& \gamma_{M I}=1 ; M_{E d}=31111111.1 \mathrm{Nmm} ; N_{c h, E d}=773991.5 \mathrm{~N} ; \\
& \left(N_{c h, E d} / N_{b, R d}\right)=773991.5 / 988762.5=0.783 \Rightarrow \\
& \left(N_{c h, E d} / N_{b, R d}\right)<1.0
\end{aligned}
$$



ments.

The chosen profile (2U24) satisfies the load-carrying require-

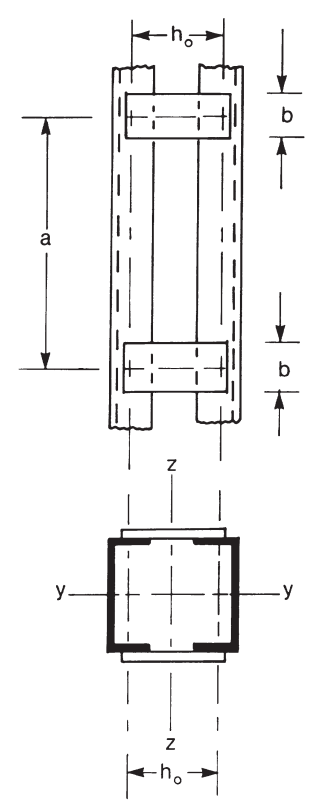

Fig. 4 Battened built up-members, (Figure 6.7, [5]).

\section{Conclusion}

By comparing the calculated normal stress to the allowable stress obtained by the $\omega$ - procedure and the standard procedure(s), it can be seen that the stresses obtained by the standard procedures are significantly smaller than stresses obtained by the theoretical procedure. This shows that the standard procedures are much more on the safety side.

The reason for this difference in stresses' values (up to $40 \%$ ) is that the calculation by the $\omega$ - procedure is done under the assumption that the material is utilized by buckling up to allowable stress, while calculations done by the standard procedures do not consider that assumption. The calculation according to Eurocode 3 is even more on the safety side than that according to standard JUS U. E7. 081. If one compares the buckling curves given by the two standards, it is not difficult to realize that they are identical. The difference in results of calculations stems from the stricter requirements by the Eurocode 3 .

\section{Acknowledgement}

This work was partially done while the first author was a visiting professor at Faculty of Civil Engineering, University of Zilina, Slovak Republic, on the SAIA grant of the Slovakian Ministry of Education.

\begin{tabular}{|c|c|}
\hline Criterion & Efficiency factor $\mu$ \\
\hline$\lambda \geq 150$ & 0 \\
\hline $75<\lambda<150$ & $\mu=2-\frac{\lambda}{75}$ \\
\hline$\lambda \leq 75$ & 1.0 \\
\hline where $\lambda=\frac{L}{i_{0}} ; i=\sqrt{\frac{I_{1}}{2 A_{c h}}} ; I_{1}=0.5 h_{0}^{2} A_{c h}+2 I_{c h}$ \\
\hline
\end{tabular}

\section{References}

[1] MILOSAVLJEVIC, M. M., et al.: Fundamentals of Steel Structures, The Civil Engineering Book, Belgrade, Serbia, 1986.

[2] OSTRIC, D.: Metal Structures, Faculty of Mechanical Engineering, Belgrade, Serbia, 1988.

[3] PETKOVIC Z., OSTRIC, D.: Metal Constructions in Machine Building - 1, Institute of Mechanization, Faculty of Mechanical Engineering, Belgrade, Serbia, 1996.

[4] JUS M.B1. GROUP OF STANDARDS: “JUS standards for construction elements, materials allowable stresses, ...”, Federal Bureau for Standardization, Belgrade, Serbia.

[5] EUROCODE 3: "Design of Steel Structures": ENV 1993-1-1: Part 1.1: General rules and rules for buildings, CEN, 1992.

[6] NIKOLIC, R., MARJANOVIC, V.: Metal Structures - Handbook for Calculations, Faculty of Mechanical Engineering, Kragujevac, Serbia, 1998.

[7] NIKOLIC, R., VELJKOVIC, J.: "The Analysis of Designing Columns Centrically Loaded by Axial Compressive Load", Proc. V Int. Conf. "Heavy Machinery”, Kraljevo, Serbia, 28-30 June, 2005, pp. IC29-IC32. 